\title{
Multi-Layer Problems and Solutions in VANETs:
}

\section{A Review}

\author{
Usman Ali Khan and Sang Sun Lee * \\ Department of Electronics \& Computers, Hanyang University, Seoul 04763, Korea; usmanali@hanyang.ac.kr \\ * Correspondence: ssnlee@hanyang.ac.kr
}

Received: 13 January 2019; Accepted: 6 February 2019; Published: 11 February 2019

\begin{abstract}
The Dedicated Short Range Communication (DSRC) technology supports the vehicular communications through Vehicle to Vehicle (V2V) and Vehicle to Infrastructure (V2I) Communication, by operating at $5.9 \mathrm{GHz}$ band (U.S. Standard). The Physical (PHY) and Medium Access Control (MAC) Layer are defined by the IEEE 802.11p, while the IEEE 1609 family of standards define the Wireless Access in Vehicular Environment (WAVE); a suite of communication and security standards in the Vehicular Area Networks (VANETs). There has been a lot of research regarding several challenges in VANETs, from spectrum utilization to multichannel operation and from routing to security issues. The aim of all is to improve the performance of the network and support scalability in VANETs; which is defined as the ability of the network to handle the addition of vehicles (nodes) without suffering noticeable degradation of performance or administrative overhead. In this paper, we aim to highlight multilayer challenges concerning the performance of the VANETs, the already proposed solutions, and the possible future work.
\end{abstract}

Keywords: DSRC; V2V; V2I; VANET; MAC; WAVE; Carrier Sense Multiple Access (CSMA); Time Division Multiple Access (TDMA); PHY; Self Organized Time Division Multiple Access (STDMA); Cognitive Radios (CR); Vehicle-to-Everything (V2X)

\section{Introduction}

Roadside accidents have increased significantly due to high traffic on highways and urban areas. In order to provide a reliable driving experience and avoiding accidents, vehicular communication was introduced to provide safety and to increase the travel experience of the user by providing on demand infotainment services. For this purpose, Wi-Fi based standard like DSRC was introduced in order to fulfill the requirements of the users for both safety and non-safety applications. While LTE based vehicular networks are being researched for the future in order to overcome the shortcomings of the DSRC.

The Dedicated Short Range Communication (DSRC) technology is being used for the safety and non-safety applications in vehicular communication. The allocated bandwidth of $75 \mathrm{MHz}$ in $5.9 \mathrm{GHz}$ spectrum band is being utilized for this purpose [1]. The IEEE Wireless Access in Vehicular Environment (WAVE) 1609 is being used as a standard for communication between vehicles. The WAVE communication model is similar to the Open System Interconnect (OSI) model with some modifications on different layer and some extensions to the management layer. Research in the past has focused on many areas from the Physical, MAC, Network, Application to the Security and Management Layers. Each Layer has its own challenges that add up to the overall performance of the DSRC. For a large number of vehicles, the congestion can severely degrade network performance which affects mostly the safety applications of the vehicles. Supporting the reliability and scalability for V2V safety communications has been the main objective of many researches in the recent past by improving the Quality of Service (QoS) parameters of the network and resolving the scalability issue. 
The DSRC architecture shown in Figure 1 includes the vehicles equipped with On Board Units (OBUs). The OBUs interact with the Road Side Units (RSUs) for the exchange of messages and applications. The RSUs are in turn connected to the external network which includes the internet cloud. The V2I communication takes place between the RSUs and OBUs for providing the internet services, weather conditions, and other infotainment applications. The V2V communication between the vehicles allows the exchange of safety messages which can avoid accidents.

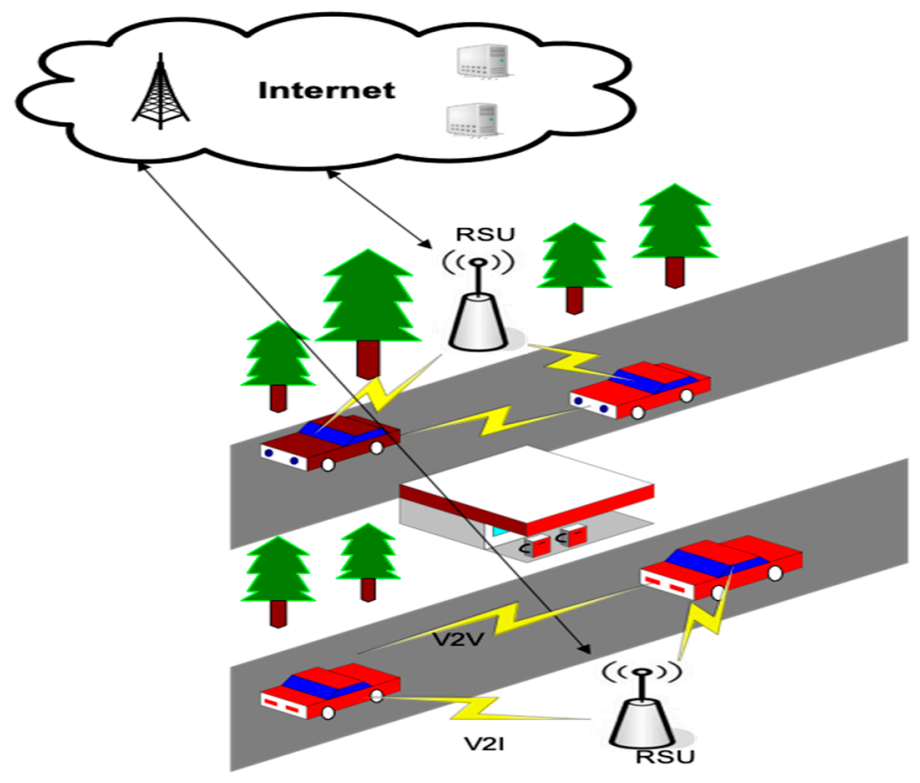

Figure 1. Dedicated Short Range Communication (DSRC) Architecture. RSU: Road Side Units; V2I: Vehicle to Infrastructure.

Several factors influence this aforementioned communication. In this paper, we explain these factors that influence the performance of VANETs by explaining the work done on each layer, challenges at every layer, and the future of VANETs. The Physical layer has been studied in terms of channel selection and estimation schemes, diversity schemes, propagation models, and cognitive radios. Whereas the MAC layer has been thoroughly researched for Multi-Channels, contention window size adjustment, hidden/exposed nodes, contention and scheduling based algorithms, and congestion control algorithms. The Routing layer has been studied in terms of different routing protocols and the advantages of cross layer routing protocols. Finally, future research directions and ongoing research in the fields of VANETs are discussed.

The rest of the paper is structured as follow: Section 2 discusses the Multi-Layer Challenges in VANETs, along with the proposed solutions. Section 3 focuses on the current trends in research and the future work, and finally Section 4 concludes the paper.

\section{Multi-Layer Challenges in VANETs}

The Physical (PHY) and MAC Layer challenges presented in [1] explains in depth the Channel Estimation at PHY layer and behavior of Carrier Sense Multiple Access (CSMA) in high node density and potential solutions to the congestion control along with the Multi-Channel Operations in IEEE 1609.4. The performance using Single and Multiple Radios has been discussed along with some simulation results. Moreover, the paper also highlights the evolution of the DSRC from the PHY and MAC Layer perspective. Campolo, et al. [2] give a very clear picture of the Multi-Channel Communications in VANETs. It gives an overview of the WAVE Stack, the Multi-Channel Operation; its issues and the countermeasures, some open issues which are not addressed yet, and like [1] uses both the Single and Multiple Radios approach to explaining the problem. The Self Organized Time Division Multiple Access (STDMA) has been used to solve the scalability problem in VANETs and has 
shown promising results, in terms of scalability and reliability, compared with CSMA [3]. Popular research issue, analysis, and trends in VANETs are presented in [4]. This paper also gives a layered view of the various underlying challenges in VANETs. But the work has been limited to the Routing, Security, and Application Layers. Perhaps the most detailed performance analysis of V2V Communication has been performed in [5]. The author has performed detailed analysis along with simulation scenarios to prove, yet again, that STDMA outperforms CSMA in high density networks.

In less dense VANETs, several performance issues are ignored as the currently available standards and protocols offer reliable performance; however, as the network size increases, many new challenges appear. The inefficiency of certain protocols becomes evident and several trade-offs exist. In this section we will see several factors, starting from the Physical Layer and moving up to the Network Layer in the communication protocol stack. The main focus will be on the WAVE Protocols, features of the protocols, and the limitations of the currently used standards in commercial vehicular networks. The security layer has been left out intentionally as the scope of this paper is mainly the layers involved in the communication of data.

\subsection{Multi-Layer Challenges}

\subsubsection{PHY Layer}

The Physical Layer of the DSRC consists of the 802.11p OFDM which works in the range of $5.9 \mathrm{GHz}$ band (5.885-5.905) with a $10 \mathrm{MHz}$ wide channel in the WAVE. The basic data rate is almost $3 \mathrm{Mbps}$ and the default data rate is $6 \mathrm{Mbps}$. The Physical Layer is a thoroughly researched area in VANETs. From transmission power control to using multiple (or single) antennas and from channel estimation to channel selection. There are many aspects of the physical layer that contribute to the limitations imposed on the scalability of the network. The Multi-path environment makes the communication very challenging due to the multi-path delay spread and mobility. The delay spread causes frequency selective fading and mobility cause the time selective fading [1]. Non Line of Sight (NLOS) conditions result in a large delay spread due to scattering and highways have an increased Doppler spread. This leads to two different challenges: the channel estimation error and the lack of time-interleaving. The Physical layer challenges in VANETs include:

1. Single and Dual Radio: The coexistence between single and dual radio is still unclear. While dual radio has some obvious advantages, the addition of a second radio in the presence of single radios does not improve the performance of safety communication under the default scheme [2].

2. Propagation Model: VANETs operate in three different kinds of environment: rural, highway, and city. The free space model which is usually used for the highway is not very accurate as the signal goes through reflections due to surrounding. In cities there are many obstacles which cause shadowing and multipath fading; therefore, free space cannot be used to model the cities. For the rural environment there are factors like trees and hills which can cause lot of reflections. So the propagation model should consider these environments.

3. Channel Selection: An analytical and simulation study is needed for the Channel Selection at the Physical layer. A game theoretic approach to selecting the best channel and data rate is mentioned in [6].

4. Channel Estimation: In order to get an accurate Channel State Information (CSI) we need advance channel estimation techniques in VANETs.

5. Diversity Techniques: Using different diversity techniques, the effects of fading and interference can be minimized.

Apart from that many transmission parameters influence the communication, e.g., message frequency, size, transmission power, modulation schemes, and coding rate. Transmission Power and Channel Load Assessment plays a crucial role [7]. The effect of fading can be reduced by using diversity techniques. Using multiple antennas, we can improve the bit error rate (BER). 


\subsubsection{MAC Layer}

The MAC Protocol plays a crucial role in fair channel access. The scalability of VANETs also relies heavily on the MAC Protocol, so developing an efficient and reliable MAC protocol is very important. Currently the IEEE 802.11p MAC along with the 1609. Multi-Channel Operations is being used as the MAC standards for vehicular communications under the WAVE technology [4]. Most research in the recent past focus on efficiently utilizing the multi channels and the performance modeling of the EDCA Mechanism to develop an analytical model in order to study the behavior of the MAC Layer. Following things should be kept in mind while designing MAC Protocol for VANETs:

1. Bandwidth Limitation: The frequent and rapidly changing topology in the VANETs contributes to the increase in the Control Overhead. The Bandwidth limitation should be kept in mind while designing a MAC Protocol in VANETs.

2. Analytical Study of the MAC Layer: It's very important to understand the nature of the MAC Layer in order to design an efficient protocol to fulfill the performance related requirements. A lot of work has been done using the stochastic modeling [8]. The performance modeling and analysis require certain criteria to be fulfilled including saturated/unsaturated conditions, number of access categories, back off counter freezing, internal/external collisions, mobility of the nodes, and computational complexity [9]. Using the Markov Chains, we can compute the transmission and collision probabilities. The analytical model results can be compared with the simulation results. An accurate model will make the behavior of MAC understandable. Currently most of the analytical modeling has been done for the IEEE 802.11p Enhanced Distributed Channel Access (EDCA) mechanism [10-12].

3. Dynamic Adjustment of the Contention Window (CW): A node in a VANET should be able to detect the congestion of the network by the received packets sequence and should be able to adjust the CW size accordingly with the help of successful packets received [13]. A number of algorithms have been proposed in literature that are used for the Dynamic Adjustment of CW with better reception rates proved through simulation [14-16]. This adjustment of CW is closely related to the Congestion Control as a default strategy to avoid congestion in CSMA/CA.

4. Congestion Control: The Decentralized Congestion Control (DCC) can work as a Cross Layer Approach by adjusting the Physical layer parameters by using the MAC layer as a channel sensing mechanism. Several Congestion Control algorithms exist for MANETs but they are not very well suited for VANETs due to the dynamic nature of network. The congestion control solutions provide high throughput (it is important to note here that for urgent safety messages, a fraction of bandwidth should be left, i.e, keep the channel load below a certain threshold). There are two main types of congestion control algorithms: Proactive and Reactive. Although now the third type known as the Hybrid Algorithms are also being researched. The key element in designing congestion control algorithms is fairness which means that the vehicles in the vicinity of each other should participate in controlling the congestion. The congestion control algorithms focus on the transmission power adjustment and the message rate adjustment [7]. In the next section we will discuss these algorithms.

5. Synchronization: Synchronization in VANETs is in a centralized manner. Currently the Universal Time Coordination (UTC) is used for the Synchronization purpose. Distributed synchronization strategies are needed along with the centralized synchronization between nodes in order to coordinate in the case of system wide failure which may result in the unavailability of the Global Clock [17].

6. Efficient Utilization of the Multi Channels: The Multi-Channel Operation was introduced in IEEE 1609.4 [18]. Currently there are 7 channels, which include the service channel (SCH) and the control channel $(\mathrm{CCH})$. The control channel is responsible for the safety messages, whereas the service channel is for the non-safety applications. A synchronization interval (Sync Interval) includes one service and one control channel. The channels shift in operation to provide 
continuous and alternating access. It is important here that the channels are utilized in the most efficient way in order to support the urgent safety messages. Lee, et al. [19] proposed a scheme to efficiently utilize the SCH which enables the service providers to be able to broadcast their services to as many vehicles as possible. The work also highlights the hidden terminal problem that can occur in a broadcast transmission in multi-channel environment.

7. Contention versus Scheduling: Using Time Division Multiple Access instead of CSMA has shown performance improvement in several studies mentioned in the next section.

8. Hidden and Exposed Terminal Problems: The Hidden and Exposed Node problem is perhaps one of the most challenging issues related to the VANETs due to the broadcast nature of the IEEE 802.11p Control Channel (CCH). The Hidden and Exposed Node Problem is shown in the Figure 2.

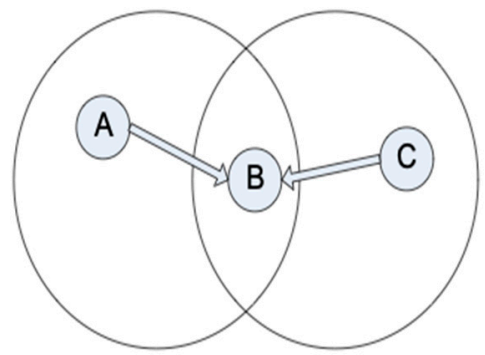

(a)

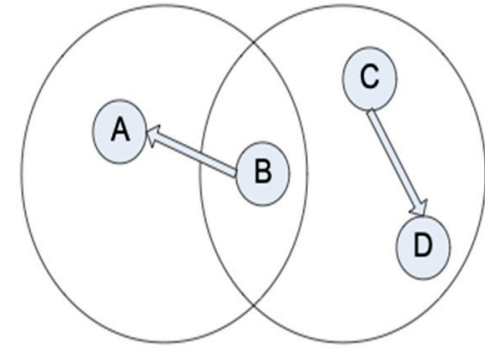

(b)

Figure 2. (a) The Hidden and (b) Exposed Terminal Problem.

As shown in the Figure 2a, the hidden terminal problem occurs when node $B$ is in the range of both nodes $A$ and $C$ but node $A$ and $C$ are not in the range of each other, so both can send data to node $\mathrm{B}$ and the collision is unavoidable as both cannot hear transmission of each other. In exposed terminal problem, shown in Figure 2b, B sends data to A, C can hear the transmission from B and prevents its transmission to $\mathrm{D}$, even if it can send the data to $\mathrm{D}$ without any problem. $\mathrm{C}$ is therefore the exposed node [20]. The MAC protocol should be designed in order to avoid this problem as it can affect the performance of the network and thus limits the scalability.

9. Distributed versus Centralized Approach: Since the VANETs are decentralized in nature, the MAC Protocol should provide good channel access, however the concepts of a centralized network can be applied to VANETs such as Clustering. Clustering based MAC protocols can be used in this regard. A few cluster based VANETs have been proposed with a cluster head $(\mathrm{CH})$ acting as a centralized node [21-23]. The cluster head is responsible for synchronization between the nodes. For a large scale network, several cluster heads exist and a rapidly varying nature of the network can put a limitation on this.

\subsubsection{Routing Layer}

Routing plays a very important role in the overall performance of any network. The optimum routing protocol sends the data through a series of hops using the shortest and less congested route. In VANETs the condition of the network has to be taken into consideration before forwarding the data as nodes join and leave the network frequently, the topology is unpredictable, and sometimes dedicated path is not available. The conventional routing algorithms does not suit the vehicular communication; thus it imposes the limitation on the performance of the network. The protocol should be capable of performing localized operations where routing decisions are purely based on information available in its vicinity. It eliminates the need for the node to know the entire topology of the network and thus it reduces the control overhead [24]. The routing layer challenges include the:

a. Route data management. 
b. Portioning of the network.

c. Variable density of the network.

An ideal routing protocol for VANETs should have the following features:

1. Neighborhood Discovery.

2. Data Forwarding Capability.

3. Geographical Information.

4. Predict the future position of vehicles.

5. Consider the uneven vehicular density.

\subsection{Proposed Solutions}

\subsubsection{PHY Layer}

1 Single versus Dual Radio: Single Radio can be used for both the safety and non-safety exchange of data. It has the benefit of being low cost and simple but suffers from poor channel utilization compared to Dual or Multi radio. With dual radio, we can use one radio for safety and other for non-safety data. The dual antenna has the advantage of better spectrum utilization, fast, and reliable delivery of real time safety data [25]. The obvious drawback of using a dual antenna is higher complexity and the cost of deployment.

2 Channel Estimation Techniques: The author in [26] proposed an inter-vehicle cooperative channel estimation method. It presents an interpolation assisted channel estimation technique to obtain the accurate Channel State Information (CSI) and mitigate the effects of imperfect CSI. The performance of channel estimation can be improved by set of different time, frequency and spatial observations. Some research in this domain is required. In [27], a Fuzzy-Based Channel Selection for Location Oriented Services in Multichannel is presented. Interference and delay are taken into consideration in order to select transmission channel in order to reduces traffic imbalance problem among the service channels. The said scheme increases throughput and offers a lower end-to-end delay compared to older techniques.

3 Diversity Techniques: The use of antenna diversity techniques such as the Multiple Input Multiple Output (MIMO) can increase the range of communication through beam forming, spatial diversity and high throughput. However, there are certain challenges of applying the MIMO technology to the vehicular environment. El-Keyi, et al. [28] mention the impact of cost and unsuitability of 802.11n for dynamic wireless channel encountered in V2V scenarios. The MIMO in V2V scenario will need channel modeling, estimation, and cross layer optimization. The benefits of diversity techniques include range extension, increased data rate, security, and reliability.

4 Software Defined Cognitive Radios: Several research in recent past has focused on using the software defined Cognitive Radios (CR) for the VANETs. The most prominent feature of CR includes their spectrum sensing ability which can enhance the efficient utilization of the spectrum. Spectrum scarcity is a major shortcoming in VANETs. However, deploying the CR in VANETs comes with its own challenges due to the dynamic nature of network, with high node mobility and unpredictable nature. A comprehensive survey of using the CR in VANETs is provided in [29]. This paper discusses the challenges of both the CR and VANETs individually and then the collective challenges when both technologies are merged. Furthermore, the paper also mentions the spectrum sensing, MAC protocols, security, and routing perspectives of the CR based VANETs. 
In Table 1 a list of Physical layer challenges is presented with some solutions along with references.

Table 1. Physical Layer.

\begin{tabular}{ccc}
\hline Challenges & \multicolumn{2}{c}{ Solutions } \\
\hline Single/Dual Antenna & $\begin{array}{c}\text { Dual Antenna for better spectrum utilization at the } \\
\text { cost of higher complexity and cost of deployment. }\end{array}$ & {$[25]$} \\
\hline Channel Selection and Estimation & $\begin{array}{c}\text { Mitigation of the effects of imperfect } \\
\text { Channel State Information }\end{array}$ & {$[26,27]$} \\
\hline Communication Range & Antenna Diversity (MIMO) & {$[28]$} \\
\hline Spectrum Scarcity & Cognitive Radios & {$[29]$} \\
\hline
\end{tabular}

\subsubsection{MAC Layer}

1 Synchronization: In the last section we discussed about the need of distributed time synchronization techniques. A comprehensive study of synchronization in VANETs is performed in [30]. The paper explains that the current synchronization algorithms are not suitable for VANETs. It also presents a new hybrid synchronization algorithm based on a whole new architecture which integrates the sensor and vehicular nodes to form a hybrid network (sensor nodes are static roadside equipment in this scenario). This integration of Wireless Sensor Networks (WSNs) with VANETs gives way to the Hybrid Clock Synchronization (HCS). The simulation proves that the algorithm is stable for both high node mobility and low traffic conditions. Research is still required for other distributed synchronization algorithms for VANETs.

2 Congestion Control Techniques: The Congestion Control is one of the most researched areas in VANETs. Figure 3 depicts the basic strategy of congestion control mechanism. The channel is sensed over a period of time and if the channel usage is above a certain threshold, the congestion control algorithm is applied and the channel is sensed again to check the impact of the algorithm. Three basic parameters are used for the congestion control: the transmission power control, packet transmission frequency, and the scheduling of messages in various channels based on priorities. A number of optimization techniques have been applied in the past to solve the congestion problem. One such technique in [31] is a meta-heuristic approach based on Tabu search algorithm for congestion control. The proposed algorithm improves the performance in terms of delay and packet loss, under varying traffic density. The impact of decentralized congestion control on contention based forwarding in VANETs is highlighted in [32]. The Decentralized Congestion Control (DCC) has been surveyed in [33] for the periodic beacon broadcast. It presents a detailed study on the classification of DCC schemes for the V2V safety communication. A dynamic congestion control scheme (DCCS) is presented in [34] whereby the control channel is monitored using self-originated event-based detection and neighbor-originated event-based detection along with adaptive congestion control. The aforementioned method has shown to improve performance in terms of low delay, lesser packet loss, and high throughput. 


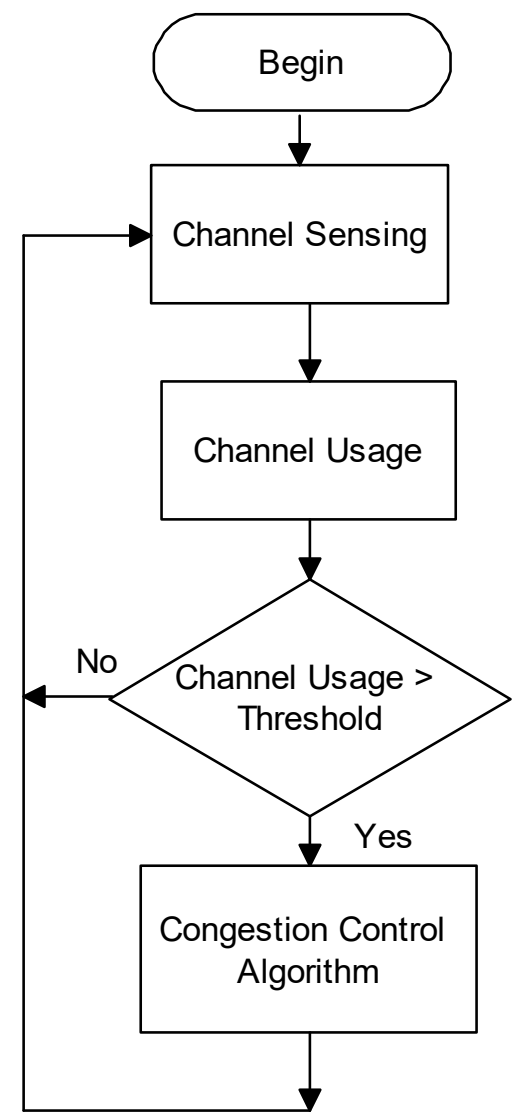

Figure 3. Congestion Control Mechanism.

The congestion control algorithms are broadly categorized in three categories:

a. Proactive Congestion Control: The proactive algorithms work by estimating the transmission parameters on the basis of number of nodes in the neighborhood. It is an open loop strategy and as the name suggest, the Proactive Congestion Control involves preventing the congestion before it can happen. The famous proactive algorithm is the Distributed Fair Power Adjustment in Vehicular Ad hoc Networks (D-FPAV) [35]. It can achieve the Congestion Control by adjusting the transmission power while the node observes the vehicles in its vicinity. In [33] the author made a performance analysis to compare the static and dynamic D-FPAV with dynamic D-FPAV showing better performance.

b. Reactive Congestion Control: The Reactive Algorithms employ the information of channel conditions and then decide how to control the congestion by tuning the transmission parameters. These algorithms sense the channel periodically, measure certain parameters, and perform a comparison with a predefined threshold. It is a closed loop strategy [36] and comes into play only after the congestion is detected in the network. It includes the Power or Rate based Congestion Control [37].

c. Hybrid Congestion Control: The Hybrid strategies combine the advantages of both Proactive and Reactive Algorithms.

3 Scheduling based MAC Protocol: The author in [38] has performed an intensive survey on the use of TDMA protocol in VANETs. There are two approaches for the TDMA protocol: Distributed and Centralized. The Distributed approach includes the Self Organized TDMA which has been extensively researched in the past for its good performance in VANETs. The Centralized approach includes the Cluster Head and the RSU. In this approach, a central node is involved in communication. The central node act as a master and communication is managed by it. 
The Scheduling based protocols like TDMA offer high throughput, low collision rate, bounded access delay, fairness and efficient utilization of channel compared with Contention based protocols. Furthermore, Hadded, et al. [38] also discusses the different protocols under each approach with their pros and cons. Nine different TDMA variants exist for a fully distributed VANET and eight different variants for the Cluster based centralized approach. These protocols perform well in different scenarios and their performance depends on several factors including the topology of the network. The STDMA has been presented in several of the recent research papers $[5,39,40]$. The STDMA comes under the category of fully distributed VANET. A scalability comparison of STDMA with the contention based CSMA of IEEE 802.11p is performed in [3]. The results show that STDMA outperforms the CSMA even in unsaturated traffic conditions. Other works include the Stabilization Time Comparison of STDMA with CSMA [41], Hidden Terminal Problem when using CSMA and STDMA [42], and Prediction based STDMA [43]. There is still a large room for research on performance comparison between the TDMA variants for vehicular communication. However, the STDMA has not been standardized as an industrial standard for VANETs.

4 Hidden and Exposed Node: In order to solve the hidden node problem, some protocols have been proposed like the Multiple Access Collision Avoidance (MACA) and MACA for Wireless (MACAW) [44]. They use the Request to Send (RTS) and Clear to Send (CTS) packets to avoid collision. When a node wishes to send data to another node, it sends an RTS message. The receiver node sends a CTS message in response and communication is established between the two nodes. During communication the other nodes in the vicinity defer to transmit. This solves the hidden node problem. However, MACA suffers from exposed node problem and thus MACAW is used. The MACAW has an additional control packet, called the Data Sending (DS) packet. This packet makes the neighboring (exposed) node aware of time duration for transmission shown in Figure 4 Other protocols to solve this problem include the Floor Acquisition Multiple Access (FAMA) and Busy Tone Multiple Access (BTMA) [44]. While this solution sounds lucrative, it is, however only good for the unicast transmission. In broadcast scenarios, RTS/CTS handshake cannot be used because of frequent collisions. The broadcast nature of IEEE 802.11p CCH for the periodic Beacon transmission, impose a big challenge to VANETs in terms of reliability and latency [33].

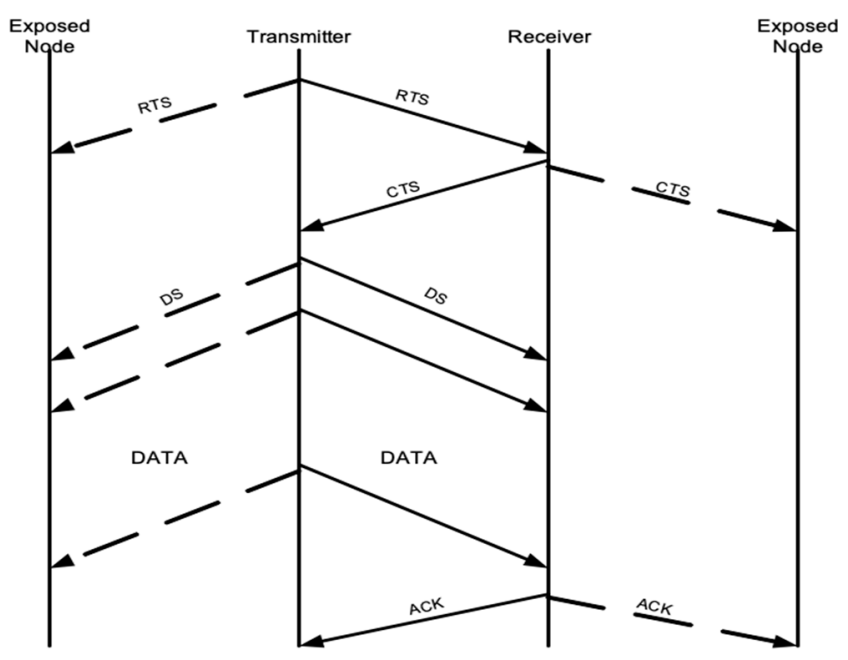

Figure 4. Exposed Node Solution in Multiple Access Collision Avoidance for Wireless (MACAW).

5 Contention Window size adjustment: One way of improving the performance is through the dynamic adjustment of the contention window. In order to provide a QOS in different application's access categories, a fuzzy logic control based dynamic adjustment of the contention window is presented in [45]. The proposed non-linear control scheme enhances the control of 
wireless access in VANETs under different operating conditions. The fuzzy logic based MAC outperforms the standard MAC in terms of throughput and provides effective differentiation among different applications' access categories. Research in [13] also shows better reception rates by the dynamic adjustment of CW in moderate network traffic. The DBM-ACW control scheme proposed in [46], aims at controlling the CW based on network density estimation in highway environments. The dynamic adjustment of CW is a CSMA/CA strategy to control the congestion [37].

6 Clustering based MAC Protocols: To improve the stability and scalability of VANETs, many cluster based algorithms have been proposed in recent research [47]. In this approach the network is divided in virtual groups i.e., clusters. Each cluster has a Cluster Head $(\mathrm{CH})$. The $\mathrm{CH}$ acts like the master of the group and manages all the communication and organization in its cluster. A Comparative Survey of VANET Clustering Techniques in [47] explores the design choices made in development of the clustering algorithms. It presents techniques to solve the problem of cluster head election and cluster management. A summary of all the techniques are mentioned in Table 2.

Table 2. Medium Access Control (MAC) Layer.

\begin{tabular}{ccc}
\hline Challenges & Solutions & \\
\hline Synchronization & Hybrid Synchronization Algorithms & {$[30]$} \\
\hline Congestion & $\begin{array}{c}\text { Congestion Detection and Control Algorithms: } \\
\text { Proactive, Reactive, and Hybrid }\end{array}$ & {$[31-37,48]$} \\
\hline Contention versus Scheduling & $\begin{array}{c}\text { Self-Organized Time Division Multiple Access } \\
\text { (STDMA) }\end{array}$ & {$[3,5,38-43]$} \\
\hline Hidden/Exposed Node & Using Request to Send (RTS)/Clear to Send (CTS) & {$[44]$} \\
packets
\end{tabular}

\subsubsection{Routing Layer}

1 Clustering Routing Protocols for VANETs: In [49] a comprehensive study of the routing protocols in vehicular networks is done. According to this study there are five broad categories of the routing protocols that can be applied to VANETs as shown in Table 1 with their subcategories.

Topology Based Protocols: There are 2 types of topology based protocols. Both use the link information to forward the traffic from one node to the next node in the network.

- Reactive: The reactive protocols reduce the congestion and overhead in network. It is an on-demand protocol and works on the requirement of the network. The main problem with this protocol is the flooding of the network due to new route discovery messages.

- Proactive: Uses the routing table to store routes. The advantage of using this protocol is low latency. But keeping the paths updated is a problem due to the fast movement of vehicles in VANETs. A proactive approach in VANETs means a lot of overhead traffic to keep the paths updated, and even so, many times, when you need the path, it is not available any more.

Geographical (Positional) Based Protocols: These protocols use the geographical or positional information of the nodes along with its neighbors using GPS and maps. These protocols are further divided into three sub-categories: DTN (Delay tolerant networks), Non-DTN and a Hybrid (DTN+Non DTN). DTN is an opportunistic routing protocol due to end to end disconnection. It is suited for applications with high latency, low data rate, and long queuing delay. Geographic based routing in VANETs have been investigated in [50] in terms of segment vehicle, link quality, and degree of 
connectivity. It uses a geographic distance routing protocol with improvement in results in terms of lower link disconnection and end-to-end delay with higher throughput compared to other state of the art protocols. Location error resilient geographical routing protocol (LER-GR) [51] has been used to improve the accuracy of the location of the vehicle which has been ignored in the past. An error calculation and location prediction with correction has been utilized to predict the location of the neighboring vehicles, which is then used for the routing purpose.

Opportunistic Routing Protocols: Assisted Data Delivery (VADD) [52] is one such protocol in which a moving vehicle carries the packet and forwards it to the first available neighbor. VADD has many different types as shown in Table 3. A Trajectory-Driven Opportunistic protocol for vehicular-cyber physical systems (VCPS) in [53], aims to lower the routing overhead compared to other opportunistic protocols.

Table 3. Routing Protocols.

\begin{tabular}{ccc}
\hline Type & Sub Category & Protocols \\
\hline \multirow{2}{*}{ Topology Based Routing Protocols } & Reactive & DSR, TORA, AODV \\
\cline { 2 - 3 } & Proactive & FSR \\
\cline { 2 - 3 } $\begin{array}{c}\text { Geographical or Position based } \\
\text { Routing Protocols }\end{array}$ & DTN & DTN-VADD, GeOpps \\
& $\begin{array}{c}\text { Non-DTN (Non-Beacon, } \\
\text { Beacon, Hybrid) }\end{array}$ & $\begin{array}{c}\text { CBF } \\
\text { PBR-DV, GPSR, GRANT, STBR, } \\
\text { CARVR, GPCR, GyTAR, } \\
\text { STAR, Gpsrj + TO - GO }\end{array}$ \\
\cline { 2 - 3 } Opportunistic Protocols & Hybrid & GeoDTN + NAV \\
\hline Interference Aware Protocols & VADD & D-VADD, L-VADD, H-VADD \\
\hline Information Dissemination & - & DUAR, SIR-ADDV, MIMO DV \\
Protocols & - & UMB \\
\hline
\end{tabular}

2 Congestion Control Routing: In [33], a congestion control based re-routing algorithm is proposed. It compares the shortest path AOMDV (Adhoc On-Demand Multipath Distance Vector) routing protocol with the proposed LIEMRO (Low Interference Energy-Efficient Multipath Routing protocol) optimal routing protocol. The result shows that the proposed solution has a better path selection capability and it reduces the overhead in the network. An improved GPSR (Greedy Parameter Stateless Routing) Protocol which controls Network Congestion in [54] is shown to reduce the delay and packet loss rate compared to the traditional GPSR Protocol.

3 Block Head Cluster based Routing: Routing can be performed by dividing the network in block head $(\mathrm{BH})$ and block nodes $(\mathrm{BN})$ [55]. It is similar to the clustered based network mentioned before. The BH and BN form a mesh topology. The BH sends continuous hello messages to the members in the block and updates status in the table. In this approach the AODV protocol has been modified to form a BM-AODV in which the nodes update their status to the BH. The results show that this strategy outperforms the conventional AODV protocol in terms of throughput and end to end delay.

4 Cross Layer Routing: Conventional Routing protocols have several retransmissions due to unsuccessful delivery of packets which can lead to congestion. Interference can be one other limiting factor. This can increase the delay in the network, making the routing scheme very inefficient. Using parameters from different layers (PHY and MAC) we can design efficient routing protocols by utilizing those parameters in decision making. This is called the Cross Layer design. Several research papers highlight the cross layer design for MANETs but there is limited work on cross layer approach in VANETs. Rehman, et al. [56] propose a scheme where these parameters have been used to make routing decision in VANETs. Using parameters from the 
physical layer (data rate, Signal to Interference and Noise Ratio, channel gain etc) and buffer space and retransmission count from the MAC layer, the upper layers can be optimized [24]. Nodes in the network can send feedback to the source node and on the basis of this feedback; the routing can be made more efficient. Some of the cross layer protocols include the SBRS-OLSR (Signal Strength Assessment based Route Selection- Optimized Link State Routing), MP2R (Mobility Prediction Progressive Routing), R-AOMDV (Adhoc On-demand Multipath Distance Vector with retransmission counts metric), and PROMPT (position-based delay-aware communication protocol) [49]. The selection of parameters from different layers is still in open issue in the cross layer design. Figure 5 shows the cross layer design for routing purpose.

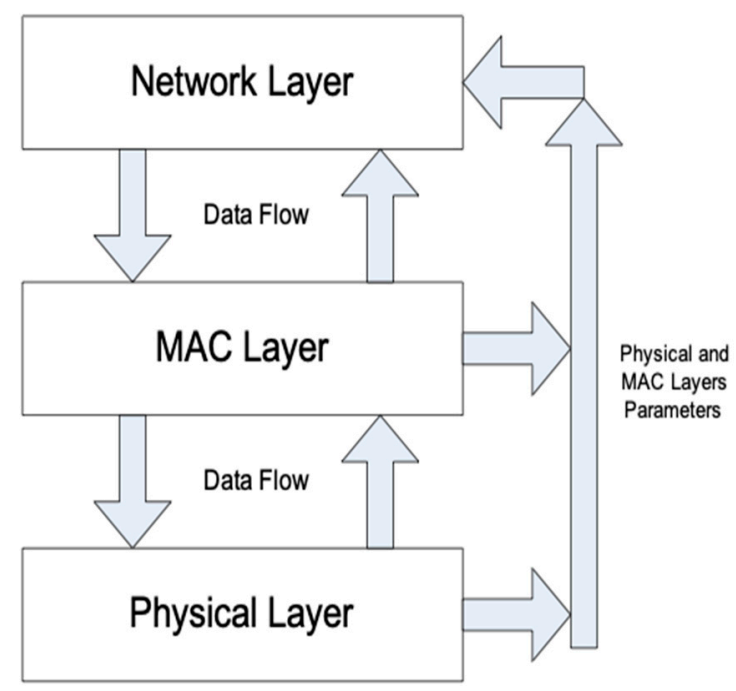

Figure 5. The Cross Layer Approach to Routing.

5 Multi-objective Dynamic Routing: Since the nature of VANETs is dynamic, dynamic routing protocols using optimization algorithms can be used to solve the routing problem. One such research [57] based on particle swarm optimization has been used to solve the multi objective problem with promising results. It considers multiple objectives like geographical ranking, customer ranking, service time, reachability time, and customer satisfaction level with constraints such as vehicle, capacity, and reachability. Geocasting based routing with particle swarm optimization in [58] has also shown good performance in terms of packet delivery and network load.

Table 4 summarizes the Routing Layer challenges and solutions.

Table 4. Routing Layer.

\begin{tabular}{ccc}
\hline Challenges & Solutions & \\
\hline Type of Protocol Used & $\begin{array}{c}\text { Topology Based } \\
\text { Geographical } \\
\text { Assisted Data Delivery } \\
\text { Dynamic Vehicle Routing Protocols }\end{array}$ & {$[25,50-53,57,58]$} \\
\hline Clustering based Protocols & Modified protocols to control the congestion & {$[54,59]$} \\
\hline Cross Layer Design & $\begin{array}{c}\text { Block Head and Block Nodes based } \\
\text { clustering protocols }\end{array}$ & {$[55]$} \\
\hline
\end{tabular}




\section{Discussion}

A lot of research had been done to in order to support a scalable VANET. Even with all the research there is still a gap between the currently used standards in industry and the research community. With the emergence of the $4 \mathrm{G}$ and $5 \mathrm{G}$ technology, the future VANETs will most likely converge with these technologies.

a. Software Defined Networks (SDN) and VANETs: The field of SDN has seen a tremendous interest by researchers in the recent past. The concept of SDN which has mainly been designed for wired networks and data centers can also be applied for the wireless networks including the VANETs. Balamurugan [60] has performed a study of using the SDN for the VANETs. It has presented network architecture, benefits of using SDN in vehicular communication, and comparison of SDN based routing with the conventional routing algorithms used in VANETs. By segregating the data and control planes of the vehicular network, it is possible to improve the overall performance of the network. The proposed architecture consists of the SDN Controller, SDN wireless node, and the SDN based RSU. The controller acts as an intelligence center and controls network behavior. The wireless node (a vehicle) and the RSU belong to the data plane. The paper also highlights the different control modes. The benefits of a software defined vehicular network will be the ease of path, power, and channel selection. It will also support a wide array of services. The Simulation from [61] also shows the routing protocol in SDN being superior to the traditional routing in VANETs (AODV, DSDV, OLSR etc). More research is needed to demonstrate the efficiency of using the SDN based VANETs that can support a scalable network and has infrastructure support. And on top of that the security aspects should also be kept in mind.

b. Cognitive Radios and VANETs: There are several open issues regarding the use of CR in VANETs. These issues include the coordination between the licensed and unlicensed network users, duration of spectrum opportunities, random movement of vehicles, multiple points of observation, interference, delay, and security [62]. The ever challenging nature of VANETs imposes many new challenges to the deployment of the CR. The CR should have the capabilities of self-optimization and adaptability to overcome these challenges. Moreover, the cost of deploying a CR in VANETs should be kept in mind.

c. Hybrid Protocols; Hybrid MAC and Routing Protocols have recently seen some interest. The main idea is to extract the advantages of one protocol in a certain situation and use another protocol for another condition. This concept can be applied to VANETs in terms of the traffic loads and topology of the network. For example, using a contention based MAC under low traffic conditions and a scheduling based MAC under heavy traffic conditions [63]. Similarly, the Hybrid Routing Protocols [64,65] are being researched in order to optimize the performance of the network and improve the throughput, end to end delay, bandwidth utilization, packet delivery ratio, and support scalability. Hybrid Congestion Control Algorithms discussed before are also being researched in order to combine the benefits of both Proactive and Reactive approaches. The complexity and additional overheads of these protocols needs further research.

d. Internet of Vehicles (IoV) and Vehicular Clouds (VCs); The fairly new concept of Internet of Vehicles (IoV) which has emerged from the Internet of Things (IoT) has captured the interest of several researchers working in the area of vehicular networks. With high speed mobile internet, there is a big room for the emerging technologies like the IoV. The traditional VANETs lack the capacity to handle the vast information available regarding other vehicles in the network. The IoV will allow the vehicles to be permanently connected to the internet to provide different services such as traffic management, road safety, and infotainment [66]. It will enable the exchange of information between vehicles, pedestrians, sensors, road side units etc. The proposed architecture of IoV is based on three layers. The first layer consists of the sensors within a vehicle, the second layer consists of the all the communication between vehicles and sensors, 
pedestrians, infrastructure, and other vehicles. It also ensures connectivity with existing and the emerging networks like the LTE and Wimax. The third layer consists of the intelligence of the IoV. This includes the storage, statistics and processing capabilities. This layer is involved in the decision making process. Different research papers have proposed different layered architectures. Currently the layered architecture of IoV is under debate and has opened gates for researchers to propose new ideas. Apart from IoV, the Vehicular Cloud (VC) is also gaining much attention recently. Ever since the internet based cloud has come under the light, there has been a tremendous research on the possibility of using the cloud computing with various networks because of the ease of storage, computing resources, and the services on the remote servers with timely availability. Therefore, the vehicular cloud can assist in the development of the intelligent transportation system (ITS). There is a need of new protocols that can aid in managing the resources that are available in vehicle and can assist in cooperation among vehicles. One such peer to peer protocol has been proposed in [67]. Clouding computing based VANETs (CC-V) are researched in [68], where the author has performed an in-depth review of the architecture and challenges of CC-V. The layered architecture, application scenarios, security and privacy and other aspects are presented. Some not so thoroughly addressed issues including data dissemination to cloud, data offloading, security and privacy aware data sharing, and data-centric routing.

e. Cooperative Multi-hop Communication: Using relay nodes in the VANETs to assist the vehicles in case of emergency or to avoid traffic jams ahead. Each vehicle in network will act like a relay node. The vehicular nodes can also contribute to self-synchronization by using a multihop communication. A new cooperative communication algorithm in case of a network failure is presented in [69]. This new algorithm known as Network Formation Game for MAC-layer retransmissions (NGOMA) reduces the delay at MAC layer due to retransmissions by selecting a suitable relay node in the vicinity to retransmit packet from a source to destination node in case of network failure. This proposed algorithm shows less transmission delays compared to non-cooperative communication. More such algorithms are needed in the future.

f. 5th Generation (5G) networks and V2X Communication: The 5G Device-to-Device (D2D) communication will be another cornerstone for the V2X communication. The D2D communication in 3GPP Release 12 is limited to providing communication in regions where network coverage is not guaranteed. Therefore, it has three coverage scenarios: in coverage, out of coverage and partial coverage [70]. Although, the mentioned scenarios are for generic mobile devices, we can extend the concept to the V2X communication. The mobile device in this case could be a moving vehicle with on-board equipment. The idea of $5 \mathrm{G}$ vehicular networks would be highly intelligent, secure, always connected, and mostly autonomous vehicles. Recently a major research trend in vehicular communication is aimed at the LTE V2X (Vehicle-to-Everything). which is based on the D2D Communication. The most important area is the resource allocation in LTE-V2X [71,72]. Various techniques have been introduced in order to solve the resource allocation problem through optimization [73,74]. The resource allocation problem has been divided into optimal power and frequency (resource blocks) assignment in order to reduce the interference from the other users. Matching [75], Graph Theory [76], and Machine Learning [77] techniques have been used in order to allocate the resources optimally. Resource allocation in LTE based vehicular networks still remains a topic of interest in the research community.

g. Cross Layer Design: As discussed in the last section, the cross layer design is an important research area in VANETs these days. Parameters from different layers are fed back to other layers and decisions are made on the basis of those parameters. Ongoing research focus on choosing these parameters in order to achieve the desired performance while preserving the modularity of the OSI architecture. 
h. Autonomous VANETs: The advances in the autonomous technology regarding the vehicular communication will facilitate in the development of intelligent transport system. The autonomous VANETs will have self-organizing, self-healing and self-optimization capabilities. In [78] the author describes the autonomic broadcast VANETs as networks with self-managed architecture, QOS based broadcasting, and optimization of broadcasting protocols. Since the broadcasting of emergency messages is an important aspect of the vehicular communication. Autonomic dissemination method (ADM) has been presented with a simulation to study the behavior of vehicles under the autonomous system. The concept of autonomous clustering has also been proposed in [79]. The concept is similar to the one mentioned in the cluster based VANETs before. A Swarm Intelligence based scalable, self-organizing, and robust Urban Public Transport System (UPTS) concept has been presented in [80].

\section{Conclusions}

In this paper we have performed a review of the factors that limit the performance in VANETs. A multi layered approach had been presented to discuss the challenges in designing the protocols and limitation of some protocols. The currently used protocols, along with new protocols have been put together and a comparison had been drawn, which paves the way to future research possibilities in the area of VANETs. Feedback techniques like Cross Layer Design and Congestion Control Algorithms are a hot topic these days. Spectrum Sensing techniques like Cognitive Radios will help ensure the efficient utilization of bandwidth. Software Defined Networks are also being researched for their possible contribution to the future VANETs. The emerging technologies in VANETs like the Internet of Vehicles (IoV) and Vehicular Clouds (VC) will act as supporting structure to the VANET Architecture. Furthermore, with the emergence of LTE-D2D technology, the vehicular communication is witnessing a shift towards the cellular communication standards, which enables more possibilities in future for research.

Funding: This research was supported by Projects for Research and Development of Police science and Technology under Center for Research and Development of Police science and Technology and Korean National Police Agency funded by the Ministry of Science, ICT and Future Planning (PA-A000001).

Conflicts of Interest: The authors declare no conflict of interest.

\section{References}

1. Wu, X.; Subramanian, S.; Guha, R. Vehicular Communication Using DSRC: Challenges, Enhancements, and Evolution. IEEE J. Sel. Areas Commun. Suppl. 2013, 31,399-408.

2. Campolo, C.; Molinaro, A. Multi-Channel Communications in Vehicular Ad hoc Networks: A Survey. IEEE Commun. Mag. 2013, 51, 158-169. [CrossRef]

3. Sjoberg-Bilstrup, K.; Uhlemann, E.; Strom, E.G. Scalability Issues of the MAC Methods STDMA and CSMA of IEEE 802.11p When Used in VANETs. In Proceedings of the IEEE International Conference on Communications Workshops, Capetown, South Africa, 23-27 May 2010.

4. Liang, W.; Li, Z.; Zhang, H.; Wong, S.; Bie, R. Vehicular Ad Hoc Networks: Architectures, Research Issues, Methodologies, Challenges, and Trends. Int. J. Distrib. Sens. Netw. 2015, 11, 745303. [CrossRef]

5. Khairnar, V.D.; Kotecha, K. Performance of Vehicle-to-Vehicle Communication using IEEE 802.11p in Vehicular Ad-hoc Network Environment. Int. J. Netw. Secur. Its Appl. (Ijnsa) 2013, 5, 143-170. [CrossRef]

6. Rawat, D.B.; Bista, B.B.; Yan, G. CoR-VANETs: Game Theoretic Approach for Channel and Rate Selection in Cognitive Radio VANETs. In Proceedings of the IEEE Seventh International Conference on Broadband, Wireless Computing, Communication and Applications, Victoria, BC, Canada, 12-14 November 2012.

7. Delgtossi, L.; Zhang, T. Vehicle Safety Communications: Protocols, Security, and Privacy, 1st ed.; John Wiley \& Sons, Inc.: Hoboken, NJ, USA, 2012.

8. Mokdad, L.; Ben-Othman, J.; Ballarini, P. Stochastic models for IEEE 802.11p. In Proceedings of the 2016 IEEE Symposium on Computers and Communication (ISCC), Messina, Italy, 27-30 June 2016. 
9. Zheng, J.; Wu, Q. Performance Modelling and Analysis of IEEE 802.11p EDCA Mechanism for VANET. IEEE Trans. Veh. Technol. 2016, 65, 2673-2687. [CrossRef]

10. Togou, M.A.; Khoukhi, L.; Hafid, A.S. Throughput Analysis of the IEEE802.11p EDCA Considering Transmission Opportunity for Non-Safety Applications. In Proceedings of the 2016 IEEE International Conference on Communications (ICC), Kuala Lumpur, Malaysia, 22-27 May 2016.

11. Shah, A.F.M.S.; Mustari, N. Modeling and Performance Analysis of the IEEE 802.11p Enhanced Distributed Channel Access Function for Vehicular Network. In Proceedings of the IEEE Future Technologies Conference 2016, San Francisco, CA, USA, 6-7 December 2016.

12. Nasrallah, Y.Y.; Al-Anbagi, I.; Mouftah, H.T. Adaptive Backoff Algorithm for EDCA in the IEEE 802.11p protocol. In Proceedings of the IEEE International Wireless Communications and Mobile Computing Conference (IWCMC), Paphos, Cyprus, 5-9 September 2016.

13. Awasthi, S.; Singh, A. Enhancement of Broadcasting in Vehicular Ad-hoc Networks (VANET) by Dynamic Adaption of Contention Window Size. Int. J. Eng. Res. Technol. (Ijert) 2013, 2, 196-200.

14. Rawat, D.B.; Yan, G.; Popescu, D.C. Dynamic Adaption of Joint Transmission Power and Contention Window in VANET. In Proceedings of the IEEE 70th Vehicular Technology Conference Fall, Anchorage, AK, USA, 20-23 September 2009.

15. Jang, H.; Feng, W. Network Status Detection-Based Dynamic Adaptation of Contention Window in IEEE 802.11p. In Proceedings of the IEEE 71st Vehicular Technology Conference, Taipei, Taiwan, 16-19 May 2010.

16. Rawat, D.B.; Popescu, D.C.; Yan, G. Enhancing VANET Performance by Joint Adaption of Transmission Power and Contention Window Size. IEEE Trans. Parallel Distrib. Syst. 2011, 22, 1528-1535. [CrossRef]

17. Morgan, Y.L. Managing DSRC and WAVE Standards Operations in a V2V Scenario. Int. J. Veh. Technol. 2010. [CrossRef]

18. IEEE Standard for Wireless Access in Vehicular Environments (WAVE)-Multi-Channel Operation, IEEE Std 1609.4-2016 (Revision of IEEE Std 1609.4-2010). Available online: https: / /ieeexplore.ieee.org/document/ 7435228 (accessed on 13 January 2019).

19. Lee, D.; Ahmed, S.H.; Kim, D. An efficient SCH utilization scheme for IEEE 1609.4 mutli-channel environemtns in VANETs. In Proceedings of the IEEE ICC 2016 Ad-hoc and Sensor Networking Symposium, Kuala Lumpur, Malaysia, 22-27 May 2016.

20. Chen, K.; Prasad, R. Cognitive Radio Network; Chapter 8; Wiley: Hoboken, NJ, USA, 2009.

21. Nasrallah, Y.Y.; Al-Anbagi, I.; Mouftah, H.T. Distributed Time Synchronization Mechanism for Large-Scale Vehicular Networks. In Proceedings of the 2016 International Conference on Selected Topics in Mobile \& Wireless Networking (MoWNeT), Cairo, Egypt, 11-13 April 2016.

22. Su, H.; Zhang, X. Clustering-Based Multichannel MAC Protocol for QoS Provisionings Over Vehicular Ad Hoc Networks. IEEE Trans. Veh. Technol. 2007, 56, 3309-3323.

23. Kim, T.; Jung, S.; Lee, S. CMMP: Clustering-Based Multi-Channel MAC Protocol in VANET. In Proceedings of the IEEE Second International Conference on Computer and Electrical Engineering, Dubai, UAE, 28-30 December 2009.

24. Hussain, K.; Awang, A.; Kamel, N.; Aissa, S. Routing in Vehicular Ad Hoc Networks: A Survey on Single and Cross-Layer Design Techniques, and Perspectives. IEEE Access 2017, 5, 9497-9517.

25. Eze, E.C.; Zhang, S.; Liu, E.; Eze, J.C. Advances in Vehicular Ad-hoc Networks (VANETs): Challenges and Road-map for Future Development. Int. J. Autom. Comput. 2016, 13, 1-18. [CrossRef]

26. Yang, Y. Inter-Vehicle Cooperative Channel Estimation for IEEE 802.11p V2I communications. IEEE J. Commun. Netw. 2017, 19, 227-238.

27. Kasana, R.; Kumar, S.; Kaiwartya, O.; Kharel, R.; Lloret, J. Fuzzy-Based Channel Selection for Location Oriented Services in Multichannel VCPS Environements. IEEE Internet Things J. 2018, 5, 4642-4651. [CrossRef]

28. El-Keyi, A.; ElBatt, T.; Bai, F.; Saraydar, C. MIMO VANETS: Research Challenges and Opportunities. In Proceedings of the IEEE International Conference on Computing, Networking and Communications (ICNC), Maui, HI, USA, 30 January-2 February 2012.

29. Lim, J.M.; Chang, Y.C.; Alias, M.Y.; Loo, J. Cognitive radio network in vehicular ad hoc networks (VANET): A survey. Cogent Eng. 2016, 3, 1191114. [CrossRef]

30. Sam, D. A Time Synchronized Hybrid VANET to Improve Road Safety. Ph.D. Thesis, Dr M.G.R Educational and Research Institute, Chennai, India, 2015. 
31. Taherkhani, N.; Pierre, S. Congestion Control in Vehicular Ad Hoc Networks Using Meta-Heuristic Techniques. In Proceedings of the Second ACM International Symposium on Design and Analysis of Intelligent Vehicular Networks and Applications, Paphos, Cyprus, 21-22 October 2012.

32. Kuhlmorgen, S.; Festag, A.; Fettweis, G. Impact of Decentralized Congestion Control on Contention-based Forwarding in VANETs. In Proceedings of the IEEE 17th International Symposium on A World of Wireless, Mobile and Multimedia Networks (WoWMoM), Coimbra, Portugal, 21-24 June 2016.

33. Song, H.; Lee, H.S. A Survey on How to Solve a Decentralized Congestion Control Problem for Periodic Beacon Broadcast in Vehicular Safety Communications. In Proceedings of the 2013 15th International Conference on Advanced Communications Technology (ICACT), PyeongChang, Korea, 27-30 January 2013.

34. Qureshi, K.N.; Abdullah, A.H.; Kaiwartya, O.; Iqbal, S.; Butt, R.A.; Bashir, F. A Dynamic Congestion Control Scheme for safety applications in vehicular ad hoc networks. Comput. Electr. Eng. Sci. Direct 2018, 72, 774-788. [CrossRef]

35. Torrent-Moreno, M.; Santi, P.; Hartenstein, H. Distributed fair transmit power adjustment for vehicular ad hoc networks. In Proceedings of the 3rd Annual IEEE Communications Society on Sensor and Ad Hoc Communications and Networks, Reston, VA, USA, 28 September 2006; pp. 479-488.

36. Taherkhandi, N. Congestion Control in Vehicular Ad hoc Networks. Ph.D. Thesis, University of Montreal, Montreal, QC, Canada, 2015.

37. Taherkhani, N.; Pierre, S. Centralized and Localized Data Congestion Control Strategy for Vehicular Ad Hoc Networks Using a Machine Learning Clustering Algorithm. IEEE Trans. Intell. Transp. Syst. 2016, 17, 3275-3285. [CrossRef]

38. Hadded, M.; Muhlethaler, P.; Laouiti, A. TDMA-Based MAC Protocols for Vehicular Ad Hoc Networks: A Survey, Qualitative Analysis, and Open Research Issues. IEEE Commun. Surv. Tutor. 2015, 17, 2461-2492. [CrossRef]

39. Yu, H.; Hc, Z.; Niu, K. STDMA for Vehicle-to-Vehicle Communication in A Highway Scenario. In Proceedings of the 5th IEEE International Symposium on Microwave, Antenna, Propagation and EMC Technologies for Wireless Communications, Chengdu, China, 29-31 October 2013.

40. Gaugel, T.; Mittag, J.; Hartenstein, H. In-depth Analysis and Evaluation of Self-Organizing TDMA. In Proceedings of the IEEE Vehicular Networking Conference, Boston, MA, USA, 16-18 December 2013.

41. Alonso, A.; Mecklenbrauker, C.F. Stabilization Time Comparison of CSMA and Self-Organizing TDMA for different channel loads in VANETs. In Proceedings of the IEEE 12th International Conference on ITS Telecommunications, Taipei, Taiwan, 5-8 November 2012.

42. Sjoberg, K.; Uhlemann, E.; Strom, E.G. How Severe is the Hidden Terminal Problem in VANETs when Using CSMA and STDMA. In Proceedings of the IEEE Vehicular Technology Conference (VTC Fall), San Francisco, CA, USA, 5-8 September 2011.

43. Verenzuela, D.; Liu, C.; Wang, L.; Shi, L. Improving Scalability of Vehicle-to-Vehicle Communication with Prediction-Based STDMA. In Proceedings of the IEEE 80th Vehicular Technology Conference, Vancouver, BC, Canada, 14-17 September 2014.

44. Sarkar, S.K.; Basavaraju, T.G.; Puttamadappa, C. Ad Hoc Mobile Wireless Networks: Principles, Protocols and Applications; Chapter 2; Auerbach Publications: Boca Raton, FL, USA, 2007.

45. Chrysostomou, C.; Djouvas, C.; Lambrinos, L. Dynamically Adjusting the Min-Max Contention Window for Providing Quality of Service in Vehicular Networks. In Proceedings of the 2012 11th Annual Mediterranean Ad Hoc Networking Workshop (Med-Hoc-Net), Ayia Napa, Cyprus, 19-22 June 2012.

46. Balador, A.; Calafate, C.T.; Cano, J.; Manzoni, P. Reducing Channel Contention in Vehicular Environments Through an Adaptive Contention Window Solution. In Proceedings of the IEEE IFIP Wireless Days (WD), Valencia, Spain, 13-15 November 2013.

47. Franklin, D.R.; Ros, M.; Safaei, F. A Comparative Survey of VANET Clustering Techniques. IEEE Commun. Surv. Tutor. 2017, 19, 657-681.

48. Nagaraj, S.R.; Nalini, N. Performance Analysis of Proactive Congestion Control Techniques for VANETs. In Proceedings of the 2016 International Conference on Wireless Communications, Signal Processing and Networking (WiSPNET), Chennai, India, 23-25 March 2016.

49. Jindal, V.; Bedi, P. Vehicular Ad-Hoc Networks: Introduction, Standards, Routing Protocols and Challenges. Int. J. Comput. Sci. Issues 2016, 13, 44. 
50. Kaiwartya, O.; Kumar, S.; Lobiyal, D.K.; Abdullah, A.H.; Hassan, A.N. Performance Improvement in Geographic Routing for Vehicular Ad Hoc Networks. Sensors 2014, 14, 22342-22371. [CrossRef]

51. Kasana, R.; Kumar, S.; Kaiwartya, O.; Yan, W.; Cao, Y.; Abdullah, A.H. Location error resilient geographical routing for vehicular ad-hoc networks. IET Intell. Transp. Syst. 2017, 11, 450-458. [CrossRef]

52. Zhao, J. VADD: Vehicle-Assisted Data Delivery in Vehicular Ad hoc Networks. IEEE Trans. Veh. Technol. 2008, 57, 1910-1922. [CrossRef]

53. Cao, Y.; Kaiwartya, O.; Aslam, N.; Han, C.; Zhang, X.; Zhuang, Y.; Dianati, M. A Trajectory-Driven Opportunistic Routing Protocol for VCPS. IEEE Trans. Aerosp. Electron. Syst. 2018, 54, 2628-2642. [CrossRef]

54. Hu, T.; Liwang, M.; Huang, L.; Tang, Y. An Enhanced GPSR Protocol based buffer length of nodes for the Congestion Problem in VANETs. In Proceedings of the 2015 10th International Conference on Computer Science \& Education (ICCSE), Cambridge, UK, 22-24 July 2015.

55. Abhilash Kumar, M.; Rama Mohan Reddy, A. A Survey on Vehicular Ad Hoc Networks Routing Protocols. i-Manager's J. Mob. Appl. Technol. 2016, 3, 19.

56. Rehman, S.; Khan, M.A.; Zia, T.A. Cross Layer Routing for VANETs. In Proceedings of the IEEE International Symposium on a World of Wireless, Mobile and Multimedia Networks 2014, Sydney, Australia, 19 June 2014.

57. Kaiwartya, O.; Kumar, S.; Lobiyal, D.K.; Tiwari, P.K.; Abdullah, A.H.; Hassan, A.N. Multiobjective Dynamic Vehicle Routing Problem and Time Seed Based Solution Using Particle Swarm Optimization. J. Sens. 2015, 2015, 189832. [CrossRef]

58. Kaiwartya, O.; Kumar, S. Geocasting in Vehicular Adhoc Networks Using Particle Swarm Optimization. In Proceedings of the International Conference on Information Systems and Design of Communication, Lisbon, Portugal, 16-17 May 2014; pp. 62-66.

59. Hassan, A.N.; Kaiwartya, O.; Abdullah, A.H.; Sheet, D.K.; Raw, R.S. Inter Vehicle Distance Based Connectivity Aware Routing in Vehicular Adhoc Networks. Wirless Pers. Commun. Int. J. 2018, 98, 33-54. [CrossRef]

60. Balamurugan, V. An Intelligent Framwork for Vehicular Networks using SDN Architecture. Int. J. Comput. Sci. Netw. 2014, 3, 435-439.

61. Ku, I.; Lu, Y.; Gerla, M.; Ongaro, F.; Gomes, R.L.; Cerqueria, E. Towards Software Defined VANET: Architecture and Services. In Proceedings of the IEEE 13th Annual Mediterranean Ad Hoc Networking Workshop (MED-HOC-NET), Piran, Slovenia, 2-4 June 2014.

62. Singh, K.D.; Rawat, P.; Bonnin, J. Cognitive Radio for Vehicular Ad hoc Networks (CR-VANETs): Approaches and challenges. Eurasip J. Wirel. Commun. Netw. 2014. [CrossRef]

63. Cao, L.; Xu, W.; Lin, X.; Lin, J. A CSMA/TDMA dynamic splitting scheme for MAC protocol in VANETs. In Proceedings of the IEEE International Conference on Wireless Communications and Signal Processing, Hangzhou, China, 24-26 October 2013.

64. Zhu, L.; Li, C.; Xia, B.; He, Y.; Lin, Q. A Hybrid Routing Protocol for 3-D Vehicular Ad Hoc Networks. IEEE Syst. J. 2017, 11, 1239-1248. [CrossRef]

65. Al-Rabayah, M.; Malaney, R. A New Scalable Hybrid Routing Protocol for VANETs. IEEE Trans. Veh. Technol. 2012, 61, 2625-2635. [CrossRef]

66. Contreras-Castillo, J.; Zeadally, S.; Guerrero-Ibanez, J. Internet of Vehicles: Architecture, Protocols, and Security. IEEE Internet Things J. 2018, 5, 3701-3709. [CrossRef]

67. Meneguette, R.I.; Boukerche, A. Peer-to-Peer Protocol for Allocated Resources in Vehicular Cloud based on V2V Communication. In Proceedings of the IEEE Wireless Communications and Networking Conference (WCNC), San Francisco, CA, USA, 19-22 March 2017.

68. Aliyu, A.; Abdullah, A.H.; Kaiwartya, O.; Cao, Y.; Usman, M.J.; Kumar, S.; Lobiyal, D.K.; Raw, R.S. Cloud Computing in VANETs: Architecture, Taxonomy, and Challenges. IETE Tech. Rev. 2018, 35, 523-547. [CrossRef]

69. Das, B.; Almhana, J. A new cooperative communication algorithm for improving connectivity in the event of network failure in VANETs. Comput. Netw. 2017, 128, 51-62. [CrossRef]

70. Schlienz, J.; Roessler, A. Device to Device Communication in LTE Whitepaper; ROHDE \& SCHWARZ: Munich, Germany, 2015.

71. Liang, L.; Li, G.Y.; Xu, W. Resource Allocation for D2D-Enabled Vehicular Communications. IEEE Trans. Commun. 2017, 65, 3186-3197. [CrossRef]

72. Sahin, T.; Boban, M. Radio Resource Allocation for Reliable Out-of-Coverage V2V Communications. In Proceedings of the 2018 IEEE 87th Vehicular Technology Conference (VTC Spring), Porto, Portugal, 3-6 June 2018. 
73. Yu, N.; Mei, J.; Zhao, L.; Zheng, K.; Zhao, H. Radio resource allocation for D2D-based V2V communications with Lyapunov optimization. In Proceedings of the 2017 IEEE/CIC International Conference on Communications in China (ICCC), Qingdao, China, 22-24 October 2017.

74. Mei, J.; Zheng, K.; Zhao, L.; Teng, Y.; Wang, X. A Latency and Reliability Guaranteed Resource Allocation Scheme for LTE V2V Communication Systems. IEEE Trans. Wirel. Commun. 2018, 17, 3850-3860. [CrossRef]

75. Gu, Y.; Saad, W.; Bennis, M.; Debbah, M.; Han, Z. Matching theory for future wireless networks: Fundamentals and applications. IEEE Commun. Mag. 2015, 53, 52-59. [CrossRef]

76. Liang, L.; Xie, S.; Li, G.Y.; Ding, Z.; Yu, X. Graph-Based Radio Resource Management for Vehicular Networks. In Proceedings of the 2018 IEEE International Conference on Communications (ICC), Kansas City, MO, USA, 20-24 May 2018.

77. Liang, L.; Ye, H.; Li, G.Y. Towards Intelligent Vehicular Networks: A Machine Learning Framework. IEEE Internet Things J. 2018. [CrossRef]

78. Mbarek, N.; Abdou, W.; Darties, B. Autonomic Computing and VANETs: Simulation of a QoS-Based Communication Model; Chapter 10, Networking Simulation for Intelligent Transportation Systems; iSTE Ltd and Wiley Publishing: London, UK, 2017.

79. Yasuharu, O.H.T.A.; Tomoyuki, O.H.T.A.; Yoshiaki, K.A.K.U.D.A. An Autonomous Clustering-based Data Trasfer Scheme Using Positions and Moving Direction of Vehicles for VANETs. In Proceedings of the IEEE Wireless Communications and Networking Conference (WCNC), hanghai, China, 1-4 April 2012.

80. Lucas, C.M.; Lopez, L.F.D.; Blas, N.G. Natural Computing Applied to the Underground System: A Synergistic Approach for Smart Cities. Sensors 2018, 18, 4094. [CrossRef] [PubMed]

(C) 2019 by the authors. Licensee MDPI, Basel, Switzerland. This article is an open access article distributed under the terms and conditions of the Creative Commons Attribution (CC BY) license (http:// creativecommons.org/licenses/by/4.0/). 